\title{
Higher power of sensorimotor rhythm is associated with better performance in skilled air-pistol shooters
}

\author{
Ming-Yang Cheng a, b, Kuo-Pin Wang ${ }^{c}$, Chiao-Ling Hung ${ }^{\mathrm{d}}$, Yu-Long Tu ${ }^{\mathrm{c}}$, \\ Chung-Ju Huang ${ }^{\mathrm{e}}$, Dirk Koester ${ }^{\mathrm{a}, \mathrm{b}}$, Thomas Schack ${ }^{\mathrm{a}, \mathrm{b}}$, Tsung-Min Hung ${ }^{\mathrm{c}}{ }^{*}$ \\ a Center of Excellence “Cognitive Interaction Technology” (CITEC), Bielefeld University, Inspiration 1, 33619, Bielefeld, Germany \\ ${ }^{\mathrm{b}}$ Neurocognition and Action - Biomechanics Research Group, Faculty of Psychology and Sports Science, Bielefeld University, Universitätsstraße 25, 33615, \\ Bielefeld, Germany \\ ${ }^{c}$ Department of Physical Education, National Taiwan Normal University, No. 162 Heping East Road Section 1, Da-an District, Taipei 106, Taiwan \\ d Department of Athletics, National Taiwan University, No. 1, Sec. 4, Roosevelt Rd., Taipei 10617, Taiwan \\ e Graduate Institute of Sport Pedagogy, University of Taipei, No.101, Sec. 2, Zhongcheng Rd., Shilin Dist., Taipei, Taiwan
}

\section{A R T I C L E I N F O}

\section{Article history:}

Received 5 December 2016

Received in revised form

31 May 2017

Accepted 31 May 2017

Available online 1 June 2017

\section{Keywords:}

Psychomotor efficiency

EEG

Sport performance

Neurofeedback

Cortical activation

Coherence

\begin{abstract}
A B S T R A C T
Objectives: Psychomotor efficiency has been linked with processing efficiency during sport performance. Reduced cortical activity in the sensorimotor area has been related to less variability in the movement preparation that is conducive to skilled motor performance. This study proposes sensorimotor rhythm (SMR), 12-15 Hz of the electroencephalography (EEG) in the sensorimotor area, may be used to investigate psychomotor efficiency in sports performance.

Method: Twenty-four skilled air pistol shooters were recruited to fire 40 shots while EEG and shooting accuracy were recorded.

Results: The data show that improved performance of skilled shooters is associated with higher SMR power during the last second and lower coherence on high alpha power at Fz-T3 before action initiation. A negative relationship is also exhibited between the SMR power and the shooting performance during the aiming.

Conclusions: This finding suggests that reduced interference from sensorimotor processing, as reflected by elevated SMR power, may be related to improved processing efficiency during the aiming period. We conclude that SMR may be used to understand psychomotor efficiency underlying air-pistol shooting performance.
\end{abstract}

(C) 2017 Elsevier Ltd. All rights reserved.
Understanding cortical processes underlying optimal performance is important for improving athletic performance. Processing efficiency as posited by the neural efficiency hypothesis refers to the general state of nervous system composed with minimal neural activation in a given task (Babiloni et al., 2010, 2008; Del Percio et al., 2011). Although neural efficiency can serve as a framework for explaining the cortical processes underlying optimal performance, recent studies of skilled self-paced performance have found that more recruitment of motor programming resources in motor-

\footnotetext{
* Corresponding author.

E-mail addresses: ming-yang.cheng@uni-bielefeld.de (M.-Y. Cheng), ben05678@ hotmail.com (K.-P. Wang), musehung@ntu.edu.tw (C.-L. Hung), c90154@hotmail. com (Y.-L. Tu), crhwang@utaipei.edu.tw (C.-J. Huang), dkoester@cit-ec.unibielefeld.de (D. Koester), thomas.schack@uni-bielefeld.de (T. Schack) ernesthungkimo@yahoo.com.tw (T.-M. Hung).
}

related areas led to superior putting performance (Cooke et al., 2014; Ring, Cooke, Kavussanu, McIntyre, \& Masters, 2015). Similarly, stronger cortical communication in the parieto-central and parieto-frontal has been found for successful putts in elite golfers (Babiloni et al., 2011). This suggests that the cortical processing in elite athletes might be more complex than that predicted solely by the "neural efficiency" hypothesis.

Psychomotor efficiency, a special case of neural efficiency, provides a more specific perspective to further understanding of the cortical processing underlying skilled self-paced performance. The psychomotor efficiency postulates less complexity in the processes associated with motor control and lower neural network activities during cognitive-motor behavior, and thus can be viewed as superior cognitive-motor processing concerning expertise (Hatfield \& Hillman, 2001, pp. 362-386). Decreased cortical activation of the motor planning-related regions (e.g. sensorimotor cortex) might 
contribute to greater consistency of the motor performance (Baumeister, Reinecke, Liesen, \& Weiss, 2008). The relationship between cortical activity and cognitive-motor processing can be investigated by using electroencephalography (EEG) activities in the sensorimotor region and the cortical communication between sensorimotor region and other regions. This direction of research has also been backed up by a general model for the interpretation of cortical activity associated with superior performance, the multiaction plan model (MAP model; Bertollo et al., 2016), which found that a silent sensorimotor area was associated with a more automated shooting performance in elite shooters.

Sensorimotor rhythm (SMR) is an ideal candidate for evaluating psychomotor efficiency in the EEG. SMR is a special category of EEG frequency ranging from 12 to $15 \mathrm{~Hz}$ observed in the sensorimotor cortex and is related to activation of this area (Sterman, 1996). Specifically, SMR power is inversely related to sensorimotor cortex activity (Sterman, 1996), indicating that lower thalamic nucleus activity is associated with less interference of somatosensory processing (Kober et al., 2015). In addition to the low and high alpha frequencies reflecting the attentional processing in general aspects and in semantic tasks, respectively (Klimesch, 1996), higher SMR power has been characterized as an adaptive state of refined taskrelated neural processing during psychomotor and attentionrelated tasks (Gruzelier, Egner, \& Vernon, 2006; Gruzelier, Inoue, Smart, Steed, \& Steffert, 2010; Kober et al., 2015; Ros et al., 2009).

In the context of sports, Cheng et al. (2015b) reported higher SMR power during the preparatory period in skilled dart-throwing players compared to novices. Furthermore, neurofeedback training (NFT) aimed at increasing SMR power resulted in improved golf putting performance (Cheng et al., 2015a). The beneficial effects of less variability in the movement preparation by augmented SMR NFT supports previous findings of lower cortical communication between Fz and T3 at high alpha range (Deeny, Hillman, Janelle, \& Hatfield, 2003), suggesting more refined processing regarding motor execution. Taken together, we propose that SMR power is potentially sensitive to complexity during motor execution, and the effect can be compared by examining cortical communication at high alpha range as it has been considered an ideal index for assessment of inter-regional communication (Von Stein \& Sarnthein, 2000). Therefore, investigating the cognitive-motor processing by using SMR power could further our understanding of psychomotor efficiency in skilled self-paced motor performance.

In this study, we used air-pistol shooting performance as the motor task because cognitive-motor processing during aiming is fundamental to skilled pistol shooting (Tremayne \& Barry, 2001). Previous studies have shown that various EEG activities can distinguish successful air-pistol shooting performance from less successful performance. For example, Loze, Collins, and Holmes (2001) found that successful air-pistol shooting performance was preceded by significantly higher occipital alpha power before trigger pulls, whereas less successful performance was preceded by reduced alpha power. Similarly, Del Percio et al. (2011) found that elite air-pistol shooters were characterized by increased cortical communication within the parietal and other posterior areas, compared to non-athletes. These authors suggest that skilled shooting performance is associated with a relatively efficient manner to process visual-spatial information. However, a more relevant EEG index which can reflect cognitive-motor processing has not been investigated in air-pistol shooting performance.

The aforementioned evidence supports the functional relation of SMR power and skilled motor performance. However, the difference in regulation of psychomotor processing during motor performance between experts and novices can be assumed to be large. The SMR differences between these two highly distinctive skill categories serve as a starting point for the relevance of SMR power in skilled motor performance. In contrast, a comparison of skilled performers' performance fluctuation represents an even more sensitive test because a trial-by-trial comparison could reveal the fine-tuning of cognitive-motor adjustment in the individual (Bertollo et al., 2013; Di Fronso et al., 2016).

Therefore, our study was designed to examine different levels of SMR power during best and worst skilled air-pistol shooting performances. Based on previous findings, we expected that lower activation of the sensorimotor cortex, as reflected by higher SMR power, would be associated with better performance.

\section{Methods}

\subsection{Participants}

Twenty-four right-handed skilled shooters (14 male; 10 female) were recruited in this study, ranging in age from 14 to 22 years old $\left(M_{\text {age }}=18.12, S D_{\text {age }}=2.39\right)$ with an average of 3.82 years ( $S D_{\text {experience }}=2.60$ years $)$ of shooting experience. They practiced shooting regularly at least four times per week. The mean shooting score of the male shooters was 557.93 and for the female shooters, 362.90. The shooters in this study were classified as B-level according to the International Sports Shooting Federation. The study was approved by an institutional review board, National Taiwan Sport University, for the protection of the human subjects. All of the participants provided their informed consent and if a participant was younger than 18 years old, a parent signed a consent form.

\subsection{Air-pistol shooting task}

To increase the ecological validity, this study adopted an actual shooting task in accordance with normal competitions instead of using an electrical shooting training system. A $10 \mathrm{~m}$ range was constructed in a purpose-built data collection building, following International Shooting Sport Federation regulations. The shooting task lasted approximately $60 \mathrm{~min}$. Four 10-shot blocks were built-in and there was a 1 min break between blocks (Deeny et al., 2003). The entire shooting session consisted of 40 self-paced shots to equalize the number of shots, as the required shots in women's shooting regulations are 40 . Participants used their own pistols to perform the shooting task to minimize unfamiliarity regarding pistol handling. Shot scores were determined by the terminal location on the target, which was a concentric circle in a $170 \mathrm{~mm} \times 170 \mathrm{~mm}$ square. The bull's eye was scored as 10 . The other eight concentric rings were marked with different diameters (an increase of $0.8 \mathrm{~cm}$ per ring) and different scores, depending on proximity to the bull's eye; a score of 9 indicated that the shot was closest to the bull's eye, and a score of 0 indicated that the shot was outside of the outermost ring but still on the target. The shot score and position for all of the participants were reported after each shot.

\subsection{EEG recording}

The EEGs were recorded from Fp1, Fp2, F7, F3, Fz, F4, F8, FT7, FC3, FCz, FC4, FT8, T3, C3, Cz, C4, T4, TP7, CP3, CPz, CP4, TP8, T5, P3, Pz, $\mathrm{P} 4, \mathrm{~T} 6, \mathrm{O} 1, \mathrm{Oz}$, and $\mathrm{O} 2$, corresponding to the International 10-10 system (Chatrian, Lettich, \& Nelson, 1985). The left and right mastoids (A1, A2) were used as an averaged ear reference for recording and offline analyses. The ground electrode was located at Fpz. For monitoring blinks and eye movements, vertical and horizontal electrooculograms (VEOG and HEOG, respectively) were recorded located superior and inferior to the right eye and on the left and right orbital canthi. EEG and EOG signals were sampled at $500 \mathrm{~Hz}$, using Neuroscan Nuamps and NeuroScan software, version 4.5 
(Neuroscan, Charlotte, NC, USA), with a band pass filter setting from 1 to $100 \mathrm{~Hz}$ and a notch filter setting of $60 \mathrm{~Hz}$. The impedance at each electrode site was maintained below $5 \mathrm{k} \Omega$. In addition, an event marker via microphone was recorded using Neuroscan software, version 4.5 , and was merged to continuous EEG recording when participants fired the shot (Hung, Haufler, Lo, Mayer-Kress, \& Hatfield, 2008). The microphone was attached to a table located at the firing line. The event markers were used in subsequent analyses to synchronize the EEG signal and behavior. The total experiment lasted approximately $1.5 \mathrm{~h}$.

\subsection{Procedures}

The participants were asked to refrain from consuming alcoholor caffeine-containing drinks for at least $24 \mathrm{~h}$ prior to performing on the shooting field. The participants were instructed on the requirements of the study and then fitted with an electrode cap (Neuroscan, Charlotte, NC, USA). Participants who were nearsighted wore their glasses or contact lens. After electrode registration, the participants were asked to practice ten warm-up shots. Subsequently, resting EEG with eyes open or closed was recorded for $60 \mathrm{~s}$ each. The participants were asked to stand firmly and to watch the target without aiming. During experimental recordings, all of the participants were asked to keep their bodies static and their eyes open for at least $3 \mathrm{~s}$ prior to shooting to minimize possible artifacts from body sway and blinking while shooting.

\subsection{Data reduction}

EOG correction (Semlitsch, Anderer, Schuster, \& Presslich, 1986) was performed on the continuous EEG data to reduce artifact signals due to blinking. A band pass of $1-30 \mathrm{~Hz}$ with a $12 \mathrm{db} /$ oct FIR filter was applied to the EEG and EOG channels. To investigate brain activity prior to initiation of the shot, EEG data were sampled starting at $3 \mathrm{~s}$ prior to the shot, based on the event markers. This segment was selected to replicate previous studies that selected the interest period before rifle, pistol, and archery performance (Del Percio et al., 2011; Doppelmayr, Finkenzeller, \& Sauseng, 2008; Hatfield, Landers, \& Ray, 1984; Haufler, Spalding, Santa; Janelle et al., 2000; Kerick et al., 2001; Loze et al., 2001). The baseline for each segment was corrected based on the entire sweep. Trials consisting of less than $3 \mathrm{~s}$ were excluded from analysis. The $3 \mathrm{~s}$ segments were further divided into three time windows, each one of which was $1 \mathrm{~s}$, that is, time window 3 (TW3: $3 \mathrm{~s}$ to $-2 \mathrm{~s}$ ), time window 2 (TW2: $-2 \mathrm{~s}$ to $-1 \mathrm{~s}$ ), and time window 1 (TW1: $1 \mathrm{~s}-0 \mathrm{~s}$ ). Artifact rejection was performed to screen for contaminated trials, which were defined by amplitudes exceeding $\pm 100 \mu \mathrm{V}$ from baseline (Baumeister et al., 2013).

To distinguish the best and worst shooting performances, the pool of ten best (scoring 9 or 10) and ten worst (scoring 8 or less) shots were selected for each participant from the remaining artifact-free trials. To preserve overall power and to transform it into spectral power $\left(\mu \mathrm{V}^{2}\right)$, fast Fourier transforms (FFTs) was used together with a Hanning windows taper ( $10 \%$ taper, $1 \mathrm{~Hz}$ bins) to retain the least spectral leakage for all artifact-free segments. A natural log-transform (Ln) was applied to ensure that the data were in normal distribution. To minimize the individual differences in EEG frequency, individual alpha frequency (IAF) and beta frequency (IBF) were performed for each subject (Del Percio et al., 2009; Klimesch, 1999). The IAF was defined as the frequency band showing the highest power in the $7.5-12.5 \mathrm{~Hz}$ spectrum. The frequency bands selected in this study were: theta (IAF-6 Hz to IAF-3 $\mathrm{Hz}$ ), low alpha (IAF-2 $\mathrm{Hz}$ to IAF), high alpha (IAF to IAF+2 Hz), SMR
$(\mathrm{IAF}+2 \mathrm{~Hz}$ to IAF $+5 \mathrm{~Hz})$. The mean alpha peak of the IAF was $9.9 \mathrm{~Hz}$ $(S D=0.9)$. The IBF was defined as the frequency band showing the highest power in the $14-30 \mathrm{~Hz}$ spectrum. The frequency bands selected in this study were: low beta (IBF- $2 \mathrm{~Hz}$ to IBF $\mathrm{Hz}$ ), and high beta as (IBF to IBF $+2 \mathrm{~Hz}$ ). The mean beta peak of the IBF was $17.7 \mathrm{~Hz}$ $(S D=2.5 \mathrm{~Hz})$.

\subsection{Data analysis}

Paired $t$-tests were conducted to determine whether the mean and standard deviation of shooting performance varied between best and worst shots. The SMR power was subjected to 2 (performance: best, worst) $\times 3$ (time window: TW3, TW2, TW1) analysis of variance (ANOVA), with repeated measures on both factors. The Greenhouse-Geisser procedure was employed to correct the degrees of freedom when the sphericity assumption was violated. Simple main effect tests were employed when the interaction effect was significant. In addition, to further test the relationship between SMR and movement variability, a correlational analysis between SMR and distance from the bull's eye was performed. The alpha level was set at 0.05. Partial eta square $\left(E t a^{2}\right)$ is reported as a measure of effect size, with values of $0.02,0.12$, and 0.26 indicating relatively small, medium, and large effect sizes, respectively (Cohen, 1992).

Four control analyses were performed to strengthen the interpretations. Age-related alpha power showed distinct patterns in participants approximately 17-18 years old in a previous study (Bresnahan, Anderson, \& Barry, 1999). To test whether the agerelated factor would show different alpha power before the shooting, participants were separated into two groups. One group contained participants who were younger than 18 years old $(\mathrm{N}=10)$; the other included those older than 18 years old $(\mathrm{N}=14)$. The alpha power was computed with 2 (age: younger than 18 years, older than 18 years) $\times 4$ (site: Fz, Cz, Pz, Oz) $\times 3$ (time window: TW3, TW2, TW1) to test this working hypothesis.

The frequency specificity test was designed to identify whether only the SMR power at $\mathrm{Cz}$ could differentiate best shots from the worst, compared to the neighboring frequency bands. The other frequency bands (i.e., theta, low-alpha, high-alpha, low-beta, highbeta) were analyzed for 2 (performance: best performance, worst performance) $\times 2$ (hemisphere: Right, Left) $\times 5$ (region: F3, F4, C3, $\mathrm{C} 4, \mathrm{~T} 3, \mathrm{~T} 4, \mathrm{P} 3, \mathrm{P} 4, \mathrm{O} 1$, and $\mathrm{O} 2$ ) $\times 3$ (time window: TW3, TW2, TW1) ANOVAs.

The EEG coherence test was designed to examine whether the best and worst shots were affected by cortico-cortical communication based on $\mathrm{C} z$. We applied Fisher's $Z$ transformation on $12-15 \mathrm{~Hz}$ to normalize the distribution of coherence in the shooting task (Deeny et al., 2003). The coherences of $12-15 \mathrm{~Hz}$ for pairs between $\mathrm{Cz}$ and $\mathrm{Fz}, \mathrm{T} 3, \mathrm{~T} 4, \mathrm{Pz}$ and $\mathrm{Oz}$ were subjected to 2 (performance: best, worst) $\times 3$ (time window: TW3, TW2, TW1) $\times 5$ (coherence sites: $\mathrm{CZ}-\mathrm{Fz}, \mathrm{CZ}-\mathrm{T} 3, \mathrm{CZ}-\mathrm{T} 4, \mathrm{Cz}-\mathrm{Pz}, \mathrm{Cz}-\mathrm{Oz}$ ) three-way ANOVA.

Cortico-cortical communication in high alpha power has been considered an index of psychomotor efficacy (Zhu, Poolton, Wilson, Maxwell, \& Masters, 2011) and characteristic of top-down activities (Von Stein \& Sarnthein, 2000) in which lower coherence between the frontal area and left temporal area indicates more efficient motor programming in movement execution. The coherences were evaluated for the high alpha power within the F3, F4, T3, T4, C3, C4, P3, P4, O1, and $\mathrm{O} 2$ electrode sites paired with Fz. A 2 (performance: best, worst) $\times 3$ (time window: TW3, TW2, TW1) $\times 10$ (coherence sites: Fz-F3, Fz-F4, Fz-T3, Fz-T4, Fz-C3, Fz-C4, Fz-P3, Fz-P4, $\mathrm{Fz}-\mathrm{O} 1, \mathrm{Fz}-\mathrm{O} 2) \times 2$ (hemisphere: left, right) four-way ANOVA was performed. 


\section{Results}

\subsection{Shooting performance}

Regarding mean shooting score, the overall average score in this study was 8.68 ( $S D=0.38)$. The average score of the 10 best shots was $9.77(0.22)$; and $7.61(0.61)$ for the 10 worst shots. The paired $t$ test showed that the average score for the 10 best shots was significantly higher than that of the 10 worst shots, $t(23)=21.5$, $p<0.001, \eta_{p}^{2}=0.950$. In regards to the consistency of shooting scores, the standard deviation of the best shots was less than that of the worst shots, $t(23)=5.282, p<0.001, \eta_{p}^{2}=0.548$.

\subsection{SMR power between best and worst shots}

Two-way ANOVA with the factors of performance (2) and time window (3) revealed a significant interaction effect, $F(2,46)=3.224$, $p=0.049, \eta_{p}^{2}=0.123$. Post hoc testing showed that SMR power was significantly higher in the best shots compared to the worst shots only at TW1, $t(23)=2.217, p=0.037, \eta_{p}^{2}=0.176$. Furthermore, the simple main effect on time window comparison in best shots was significant, $F(2,46)=7.827, p<0.001, \eta_{p}^{2}=0.385$. The post hoc test showed that significantly higher SMR power was observed at TW1 than at TW2, $t(23)=4.568, p<0.001, \eta_{p}^{2}=0.476$ and TW $3, t(23)=4.407, p<0.001$, $\eta_{p}^{2}=0.458$. The result of this part is illustrated in Fig. 1 .

\subsection{Correlation between SMR and distance from the bull's eye}

The average distance from the bull's eye and SMR power were $1.22(0.19) \mathrm{cm}$ and $0.86(1.07) \mu \mathrm{V}^{2}$, respectively. The Pearson's correlation analysis showed that the SMR power during $3 \mathrm{~s}$ before shooting was negatively correlated with the average distance from the bull's eye ( $r=-0.468, p=0.021, N=24)$.

\subsection{Control analysis}

\subsubsection{Age-related alpha power}

Age $(2) \times$ site $(4) \times$ time window (3) three-way ANOVA showed neither the interaction effect, $F(6,132)=0.173, p=0.984$, $\eta_{p}^{2}=0.008$, nor a main effect of the age factor, $F(1,22)=0.459$, $p=0.505, \eta_{p}^{2}=0.020$, on alpha power.

\subsubsection{Frequency specificity}

The separate performance $(2) \times$ hemisphere $(2) \times$ region (5) $\times$ time window (3) ANOVAs showed no interaction effect for theta, $F(8,184)=0.728, p=0.450, \eta_{p}^{2}=0.225$; low alpha, $F(8$, $184)=0.676, p=0.488, \eta_{p}^{2}=0.142$; high alpha, $F(8,184)=0.423$, $p=0.628, \eta_{p}^{2}=0.478$; low beta, $F(8,184)=0.308, p=0.637$, $\eta_{p}^{2}=0.253$, and high beta, $F(8,184)=0.316, p=0.669, \eta_{p}^{2}=0.145$.

\subsubsection{SMR coherence between best and worst shot performances}

The performance $(2) \times$ time window $(3) \times$ coherence $(5)$ threeway ANOVA showed neither an interaction effect, $F(8,184)=0.655$, $p=0.730, \eta_{p}^{2}=0.028$ nor an interaction of performance $(2) \times$ time window (3), $F(2,46)=1.389, p=0.260, \eta_{p}^{2}=0.057$, or performance (2) $\times$ coherence $(5), F(4,92)=0.823, p=0.514, \eta_{p}^{2}=0.035$.

\subsubsection{The coherence of high alpha power and shooting performance}

The performance $(2) \times$ time window $(3) \times$ coherence (10) $\times$ hemisphere (2) four-way ANOVA showed a significant performance $\times$ coherence site interaction, $F(4,92)=2.719$, $p=0.034, \eta_{p}^{2}=0.106$. Post hoc analysis indicated that the best performance $(M=0.396, S D=0.035)$ exhibited significantly lower coherence, $t(23)=2.378, p=0.026, \eta_{p}^{2}=0.197$, on high alpha power at Fz-T3 than the worst performance $(M=0.468, S D=0.038)$.

\section{Discussion}

To our knowledge, this is the first study to empirically investigate the SMR power among intra-individual trials in sports, especially for action execution. Our results showed that best shooting performance was preceded by higher SMR power during the last second before action initiation. Furthermore, SMR power was negatively correlated with shooting performance (distance from the bull's eye). Both findings provide support for the relevance of SMR power in cortical processes underlying superior shooting performance and in support of the concept of psychomotor efficiency. Notably, the consideration concerning age-related difference on SMR power has been ruled out in our analysis.

The association of lower activation in sensorimotor cortex as reflected by higher SMR power supports psychomotor efficiency in superior shooting performance. Previous studies have suggested that higher SMR power is correlated with lower activity of the sensorimotor cortex (Sterman, 1996) and lower activation in this area has been associated with various positive effects on skilled motor performance. For instance, Ros et al. (2009) found that a shortened overall operation duration and lower anxiety score were observed following augmented SMR NFT in microsurgery performance. Gruzelier et al. (2010) also revealed that improved acting performance and a higher subjective flow state were reported with augmented SMR power after NFT. These results suggest that augmented SMR power is related to improved attention-related processes through fine-tuning impulse control, as well as the integration of relevant environmental stimuli. This explanation is supported by previous studies of the positive effects of augmented SMR NFT on cognitive performance. For example, Egner and Gruzelier (2001) observed, after SMR NFT, an increased P300b amplitude at frontal, central, and parietal sites in the auditory oddball task, as well as reductions in commission errors and reaction time variability on the Test of Variables of Attention. Similarly, Doppelmayr and Weber (2011) demonstrated that participants exhibited improved performance on spatial rotation, simple, and choice reaction time tasks, suggesting advanced visuospatial ability after augmented SMR NFT. Augmented SMR NFT also improved the memory and attentional performance, accompanied by increased N1 and P3 event-related potential amplitudes (Kober et al., 2015). In the context of sport performance, higher SMR power in the final

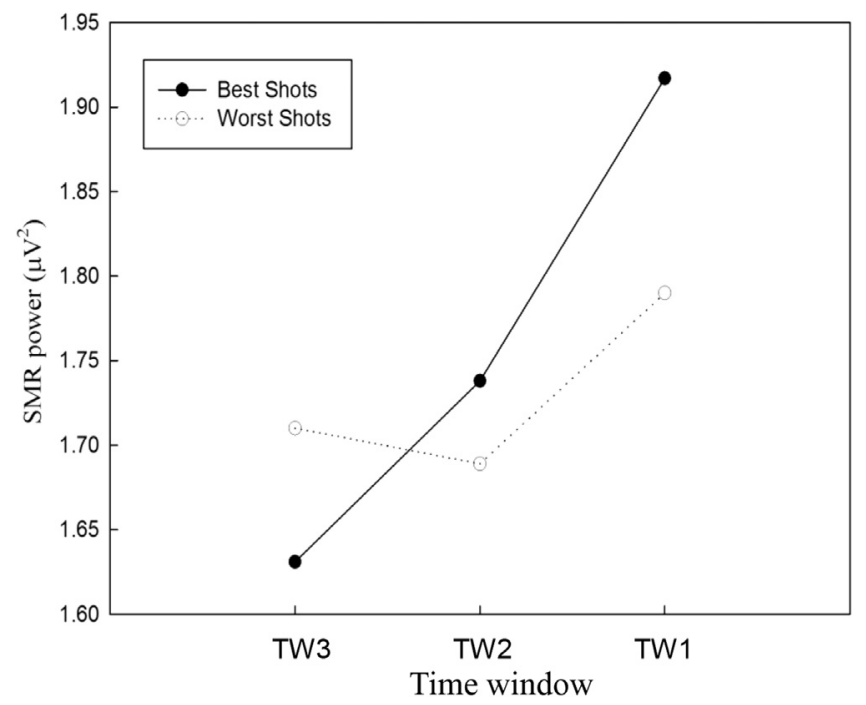

Fig. 1. The SMR power between the best and worst shots $3 \mathrm{~s}$ prior to the shot on $\mathrm{Cz}$ electrode. 
phase of preparation was linked with better performance output in dart-throwing (Cheng et al., 2015b) and golf putting (Cheng et al., 2015a). Collectively, these results suggest that lower sensorimotor processing decreased interference with visual processing (Sterman, 1996), indicating a lower complexity during motor performance, which could strengthen cognitive performance by improving attentional processing (Kober et al., 2015). This adaptive cognitive processing is conducive to natural internal guidance for action execution (Milton, Solodkin, Hluštík, \& Small, 2007) and is associated with the type of optimal-automatic performance from the MAP model (Bertollo et al., 2016; Di Fronso et al., 2016).

The SMR power as a promising EEG component for distinguishing the subtle differences in cognitive processes between the best and worst air-pistol shooting performances was further supported by our control analysis. The results showed that superior shooting performance was related to higher power in $12-15 \mathrm{~Hz}$ at the sensorimotor area whereas the neighboring frequency bands remained indifferent. The frequency specificity of the SMR power in skilled motor performance is in agreement with previous studies (Cheng et al., 2015a), which suggests a negative relationship between SMR and the variability in the movement preparation and supports the notion that the sensorimotor cortex may serve as a specific brain area for processing the most relevant cognitive information during air-pistol shooting performance. The finding of no significant cortico-cortical communication between $\mathrm{Cz}$ and other electrodes along with the notion that the sensorimotor area is a key region for processing motor interference information (Kober et al., 2015; Sterman, 1996) and pure motor information (Hatfield \& Hillman, 2001, pp. 362-386) provide another support for the specific role of SMR in motor performance. The more economical activities in the somatosensory area and premotor area in movement preparation were also discovered in a previous study, which showed that more reduced brain activities were found in top-level shooters than novices in a self-paced finger tapping task (Di Russo, Pitzalis, Aprile, \& Spinelli, 2005), suggesting a more refined neural processing. Taken together, higher SMR power, representing inhibition of sensorimotor cortical activation, might lead to fine-tuned neural processing in the sensorimotor area, which results in superior shooting performance. This finding supports the notion of psychomotor efficiency.

Apart from the activity of a single EEG electrode, less irrelevant processing prior to trigger pull might result in better shooting performance. We found that the high alpha coherence was lower in $\mathrm{Fz}-\mathrm{T} 3$ before trigger pull for the best performance than the worst, suggesting that the communication between the motor planning and the verbal-analytical regions during the motor task was more fine-tuned, with greater attenuation of irrelevant cortical coactivation. Reduced verbal-analytic processing has been associated with expertise but not relevant in novices during the motor performance (Deeny et al., 2003; Hatfield, Haufler, Hung, \& Spalding, 2004; Haufler, Spalding, Maria, \& Hatfield, 2000). This result was in agreement with previous findings inferring that greater psychomotor efficiency during movement execution is based on reduced co-activation of Fz-T3 coherence (Zhu et al., 2011). The best performance in our study is consistent with the Type 1 performance state construed within the MAP model (Bertollo et al., 2016; Di Fronso et al., 2016), which suggests that less conscious control on performance is related to the optimalautomatic motor performance. Therefore, psychomotor efficiency could be considered as a part of the optimal performance categorized by the MAP model, indicating that the degree of perceived control separates the Type 1 (Efficient processing) performance from the Type 2 (Effortful processing) performance (Bortoli, Bertollo, Hanin, \& Robazza, 2012; Robazza, Bertollo, Filho, Hanin, \& Bortoli, 2016). As previous studies have shown that alpha coherence is related to top-down processing, which might converge the sensory input from multiple areas (Von Stein \& Sarnthein, 2000), our study indicates that examining SMR power is prospective and influential for understanding the psychomotor processing in precision sports (Cheng et al., 2015b, 2015a).

The finding of higher SMR in superior performance assisted in understanding lower variability in the preparation of self-paced performance. Several EEG studies have been suggested relating to this argument in precision sports. For example, previous studies have demonstrated that lower frontal midline theta power was associated with better performance in basketball free throw (Chuang, Huang, \& Hung, 2013) and golf putting (Kao, Huang, \& Hung, 2013), suggesting a more efficient cortical load during the tasks. Similarly, higher alpha power at the occipital region was associated with better air-pistol shooting performance, suggesting a state of more intentional processing instead of controlled processing (Loze et al., 2001). In addition, lower cortical communication between the frontal and left temporal regions has been found in expert marksmen compared to lesser skilled shooters (Deeny et al., 2003). Higher SMR power in superior shooting performance suggested an adaptive cortical processing with lower interference and complexity from sensorimotor processing.

Taken together, the more efficient cortical processing symbolizes a distinguishing index for superior motor performance, indicating that lesser noise and more task-related cortical processing are the fundamental factors composing peak performance. These findings and interpretation provide convergent evidence supporting the notion of psychomotor efficiency. Furthermore, the more automatic processing in the best performance as reflected by higher SMR power is consistent with the Type 1 performance state of the MAP model, suggesting a superior psychomotor efficiency performed by the shooters. Future studies should work on drawing a more comprehensive picture regarding optimal cortical processing, especially the Type 2 performance state proposed by the MAP model, in self-paced performance by combining these established EEG indices.

The understanding regarding cortical complexity of motor performance, as reflected by SMR power, could be improved by several methodological considerations. First, future studies should include some relevant subjective measurements with mental scales when designing their experiments, e.g. the Activation/DeactivationAdjectives Checklist (Gruzelier, 2014) or the flow state scale (Jackson \& Eklund, 2004). Second, registration of high-density electrodes and the use of analytical tools with high spatial resolution should be considered to be more precise about the sources of EEG signals. Third, shooting quality could be further classified based on objective measurements, such as the markers of shot 'hold' and triggering 'trace length', as well as subjective measurements, such as self-reported performance (Di Fronso et al., 2016). Fourth, the examination of functional connectivity concerning SMR activity is still warranted for future studies, especially in relation to cortical communication at the high alpha frequency band, which has been addressed as a sensitive index of inter-region communication (Von Stein \& Sarnthein, 2000). Kober et al. (2015) found that augmented SMR power might reflect sensorimotor interference, as reflected by reduced functional connectivity between motor areas and parietal-occipital areas. However, the shooters in this study exhibited identical coherences at the SMR band between best and worst shots before trigger pulls. These incongruent findings might be due to the design of the studies (e.g., between subjects vs. within subjects). The changes in network dynamics regarding SMR activity should be further investigated to close the knowledge gap regarding cortical interaction during the final phase of action execution, especially considering that the connectivity between sensorimotor cortex and parietal cortex on high alpha frequency 
bandwidth is believed to be functionally related to motor performance (Baumeister et al., 2013).

\section{Conclusion}

In summary, skilled air-pistol shooters exhibited higher SMR power during the last second before best shots than before worst shots, suggesting reduced activity in the sensorimotor cortex, possibly reflecting how inhibition of nonessential sensorimotor information input and lower variability in the movement preparation is antecedent for superior task execution. The frequency specificity and coherence analyses provided additional support for the significant activity of SMR power in air-pistol shooting performance. These findings lend support to previous evidence suggesting that fine-tuned activation in the sensorimotor cortex is essential to producing more automatic processing in skilled performance (Baumeister et al., 2008). These observations are in line with the psychomotor efficiency hypothesis and the Type 1 performance state of the MAP model (Bortoli et al., 2012; Robazza et al., 2016), which suggests that optimal performance is constructed by a more automated manner with minimal conscious on performance control.

\section{Acknowledgments}

The work of Tsung-Min Hung was supported in part by the Ministry of Science and Technology (Taiwan) under grant NSC 1032410-H-003 -113 -MY3.

\section{References}

Babiloni, C., Del Percio, C., Iacoboni, M., Infarinato, F., Lizio, R., Marzano, N., ... Eusebi, F. (2008). Golf putt outcomes are predicted by sensorimotor cerebral EEG rhythms. The Journal of Physiology, 586, 131-139. http://doi. org/10.1113/jphysiol.2007.141630.

Babiloni, C., Infarinato, F., Marzano, N., Iacoboni, M., Dassù, F., Soricelli, A., ... Del Percio, C. (2011). Intra-hemispheric functional coupling of alpha rhythms is related to golfer's performance: A coherence EEG study. International Journal of Psychophysiology, 82, 260-268. http://doi.org/10.1016/j.ijpsycho.2011.09.008.

Babiloni, C., Marzano, N., Infarinato, F., Iacoboni, M., Rizza, G., Aschieri, P., ... Del Percio, C. (2010). "Neural efficiency" of experts' brain during judgment of actions: A high-resolution EEG study in elite and amateur karate athletes. Behavioural Brain Research, 207, 466-475. http://doi.org/10.1016/j.bbr.2009.10. 034.

Baumeister, J., Reinecke, K., Liesen, H., \& Weiss, M. (2008). Cortical activity of skilled performance in a complex sports related motor task. European Journal of Applied Physiology, 104, 625-631. http://doi.org/10.1007/s00421-008-0811-x.

Baumeister, J., Von Detten, S., Van Niekerk, S. M., Schubert, M., Ageberg, E., \& Louw, Q. A. (2013). Brain activity in predictive sensorimotor control for landings: An EEG pilot study. International Journal of Sports Medicine, 34, 1106-1111. http://doi.org/10.1055/s-0033-1341437.

Bertollo, M., Bortoli, L., Gramaccioni, G., Hanin, Y., Comani, S., \& Robazza, C. (2013). Behavioural and psychophysiological correlates of athletic performance: A test of the multi-action plan model. Applied Psychophysiology Biofeedback, 38. http:// doi.org/10.1007/s10484-013-9211-z.

Bertollo, M., Di Fronso, S., Filho, E., Conforto, S., Schmid, M., Bortoli, L., ... Robazza, C. (2016). Proficient brain for optimal performance: The MAP model perspective. PeerJ, 4, e2082. http://doi.org/10.7717/peerj.2082.

Bortoli, L., Bertollo, M., Hanin, Y., \& Robazza, C. (2012). Striving for excellence: A multi-action plan intervention model for shooters. Psychology of Sport and Exercise, 13, 693-701. http://doi.org/10.1016/j.psychsport.2012.04.006.

Bresnahan, S. M., Anderson, J. W., \& Barry, R. J. (1999). Age-related changes in quantitative EEG in attention-deficit/hyperactivity disorder. Biological Psychiatry, 46, 1690-1697. http://doi.org/10.1016/S0006-3223(99)00042-6.

Chatrian, G. E., Lettich, E., \& Nelson, P. L. (1985). Ten percent electrode system for topographic studies of spontaneous and evoked EEG activity. The American Journal of EEG Technology, 25, 83-92. http://doi.org/10.1080/00029238.1985. 11080163.

Cheng, M. Y., Huang, C. J., Chang, Y. K., Koester, D., Schack, T., \& Hung, T. M. (2015a). Sensorimotor rhythm neurofeedback enhances golf putting performance. Journal of Sport \& Exercise Psychology, 37, 626-636. http://doi.org/10.1123/jsep. 2015-0166.

Cheng, M. Y., Hung, C. L., Huang, C. J., Chang, Y. K., Lo, L. C., Shen, C., et al. (2015b). Expert-novice differences in SMR activity during dart throwing. Biological Psychology, 110, 212-218. http://doi.org/10.1016/j.biopsycho.2015.08.003.
Chuang, L. Y., Huang, C. J., \& Hung, T. M. (2013). The differences in frontal midline theta power between successful and unsuccessful basketball free throws of elite basketball players. International Journal of Psychophysiology, 90, 321-328. http://doi.org/10.1016/j.ijpsycho.2013.10.002.

Cohen, J. (1992). A power primer. Psychological Bulletin, 112, 155-159. http://doi.org/ 10.1037/0033-2909.112.1.155.

Cooke, A., Kavussanu, M., Gallicchio, G., Willoughby, A., Mcintyre, D., \& Ring, C. (2014). Preparation for action: Psychophysiological activity preceding a motor skill as a function of expertise, performance outcome, and psychological pressure. Psychophysiology, 51, 374-384. http://doi.org/10.1111/psyp.12182.

Deeny, S. P., Hillman, C. H., Janelle, C. M., \& Hatfield, B. D. (2003). Cortico-cortical communication and superior performance in skilled marksmen: An EEG coherence analysis. Journal of Sport \& Exercise Psychology, 25, 188-204. Retrieved from http://journals.humankinetics.com/AcuCustom/Sitename/ Documents/DocumentItem/1288.pdf.

Del Percio, C., Babiloni, C., Bertollo, M., Marzano, N., Iacoboni, M., Infarinato, F., ... Eusebi, F. (2009). Visuo-attentional and sensorimotor alpha rhythms are related to visuo-motor performance in athletes. Human Brain Mapping, 30, 3527-3540. http://doi.org/10.1002/hbm.20776.

Del Percio, C., Iacoboni, M., Lizio, R., Marzano, N., Infarinato, F. Vecchio, F., ... Babiloni, C. (2011). Functional coupling of parietal alpha rhythms is enhanced in athletes before visuomotor performance: A coherence electroencephalographic study. Neuroscience, 175, 198-211. http://doi.org/10.1016/j. neuroscience.2010.11.031.

Di Fronso, S., Robazza, C., Filho, E., Bortoli, L., Comani, S., \& Bertollo, M. (2016) Neural markers of performance states in an Olympic athlete: An EEG case study in air-pistol shooting. Journal of Sports Science and Medicine, 15, 214-222.

Di Russo, F., Pitzalis, S., Aprile, T., \& Spinelli, D. (2005). Effect of practice on brain activity: An investigation in top-level rifle shooters. Medicine and Science in Sports and Exercise, 37, 1586-1593. http://doi.org/10.1249/01.mss.0000177458. 71676.0d.

Doppelmayr, M., Finkenzeller, T., \& Sauseng, P. (2008). Frontal midline theta in the pre-shot phase of rifle shooting: Differences between experts and novices. Neuropsychologia, 46, 1463-1467. http://doi.org/10.1016/j.neuropsychologia. 2007.12.026.

Doppelmayr, M., \& Weber, E. (2011). Effects of SMR and theta/beta neurofeedback on reaction times, spatial abilities, and creativity. Journal of Neurotherapy, 15 , 115-129. http://doi.org/10.1080/10874208.2011.570689.

Egner, T., \& Gruzelier, J. (2001). Learned self-regulation of EEG frequency components affects attention and event-related brain potentials in humans. Neuroreport, 12, 4155-4159. http://doi.org/10.1097/00001756-200112210-00058.

Gruzelier, J. (2014). EEG-neurofeedback for optimising performance. Iii: A review of methodological and theoretical considerations. Neuroscience and Biobehavioral Reviews, 44, 159-182. http://doi.org/10.1016/j.neubiorev.2014.03.015.

Gruzelier, J., Egner, T., \& Vernon, D. (2006). Chapter 27 Validating the efficacy of neurofeedback for optimising performance. Progress in Brain Research, 159, 421-431. http://doi.org/10.1016/S0079-6123(06)59027-2.

Gruzelier, J., Inoue, A., Smart, R., Steed, A., \& Steffert, T. (2010). Acting performance and flow state enhanced with sensory-motor rhythm neurofeedback comparing ecologically valid immersive VR and training screen scenarios. Neuroscience Letters, 480, 112-116. http://doi.org/10.1016/j.neulet.2010.06.019.

Hatfield, B. D., Haufler, A. J., Hung, T. M., \& Spalding, T. W. (2004). Electroencephalographic studies of skilled psychomotor performance. Journal of Clinical Neurophysiology, 21, 144-156. http://doi.org/10.1097/00004691-20040500000003.

Hatfield, B. D., \& Hillman, C. H. (2001). The psychophysiology of sport: A mechanistic understanding of the psychology of superior performance. In handbook of sport psychology. Retrieved from http://kch.illinois.edu/research/labs/neurocognitivekinesiology/files/Articles/Hatfield_2001_ThePsychophysiologyOfSport.pdf.

Hatfield, B. D., Landers, D. M., \& Ray, W. J. (1984). Cognitive processes during selfpaced motor performance: An electroencephalographic profile of skilled marksmen. Journal of Sport Psychology, 6, 42-59.

Haufler, A. J., Spalding, T. W., Santa Maria, D. L., \& Hatfield, B. D. (2000). Neurocognitive activity during a self-paced visuospatial task: Comparative EEG profiles in marksmen and novice shooters. Biological Psychology, 53, 131-160. http://doi.org/10.1016/S0301-0511(00)00047-8.

Hung, T. M., Haufler, A. J., Lo, L. C., Mayer-Kress, G., \& Hatfield, B. D. (2008). Visuomotor expertise and dimensional complexity of cerebral cortical activity. Medicine and Science in Sports and Exercise, 40, 752-759. http://doi.org/10.1249/ MSS.0b013e318162c49d.

Jackson, S., \& Eklund, R. C. (2004). The flow scales manual. Morgantown, WV: Fitness Information Technology.

Janelle, C. M., Hillman, C. H., Apparies, R. J., Murray, N. P., Meili, L., Fallon, E. A., et al (2000). Expertise differences in cortical activation and gaze behavior during rifle shooting. Journal of Sport and Exercise Psychology, 22, 167-182.

Kao, S. C., Huang, C. J., \& Hung, T. M. (2013). Frontal midline theta is a specific indicator of optimal attentional engagement during skilled putting performance. Journal of Sport \& Exercise Psychology, 35, 470-478. Retrieved from http://www. ncbi.nlm.nih.gov/pubmed/24197715.

Kerick, S. E., McDowell, K., Hung, T. M., Santa Maria, D. L., Spalding, T. W., \& Hatfield, B. D. (2001). The role of the left temporal region under the cognitive motor demands of shooting in skilled marksmen. Biological Psychology, 58, 263-277. http://doi.org/10.1016/S0301-0511(01)00116-8.

Klimesch, W. (1996). Memory processes, brain oscillations and EEG synchronization. International Journal of Psychophysiology, 24, 61-100. http://doi.org/10. 
1016/S0167-8760(96)00057-8.

Klimesch, W. (1999). EEG alpha and theta oscillations reflect cognitive and memory performance: A review and analysis. Brain Research Reviews, 29, 169-195. http://doi.org/10.1016/S0165-0173(98)00056-3.

Kober, S. E., Witte, M., Stangl, M., Väljamäe, A., Neuper, C., \& Wood, G. (2015). Shutting down sensorimotor interference unblocks the networks for stimulus processing: An SMR neurofeedback training study. Clinical Neurophysiology, 126, 82-95. http://doi.org/10.1016/j.clinph.2014.03.031.

Loze, G. M., Collins, D., \& Holmes, P. S. (2001). Pre-shot EEG alpha-power reactivity during expert air-pistol shooting: A comparison of best and worst shots. Journal of Sports Sciences, 19, 727-733. http://doi.org/10.1080/02640410152475856.

Milton, J., Solodkin, A., Hluštík, P., \& Small, S. L. (2007). The mind of expert motor performance is cool and focused. NeuroImage, 35, 804-813. http://doi.org/10. 1016/j.neuroimage.2007.01.003.

Ring, C., Cooke, A., Kavussanu, M., McIntyre, D., \& Masters, R. (2015). Investigating the efficacy of neurofeedback training for expediting expertise and excellence in sport. Psychology of Sport and Exercise, 16, 118-127. http://doi.org/10.1016/j. psychsport.2014.08.005.

Robazza, C., Bertollo, M., Filho, E., Hanin, Y., \& Bortoli, L. (2016). Perceived contro and hedonic tone dynamics during performance in elite shooters. Research Quarterly for Exercise and Sport, 87. http://doi.org/10.1080/02701367.2016.
1185081.

Ros, T., Moseley, M. J., Bloom, P. A., Benjamin, L., Parkinson, L. A., \& Gruzelier, J. (2009). Optimizing microsurgical skills with EEG neurofeedback. BMC Neuroscience, 10(87). http://doi.org/10.1186/1471-2202-10-87.

Semlitsch, H. V., Anderer, P., Schuster, P., \& Presslich, O. (1986). A solution for reliable and valid reduction of ocular artifacts, applied to the P300 ERP. Psychophysiology, 23, 695-703. http://doi.org/10.1111/j.1469-8986.1986.tb00696.x.

Sterman, M. B. (1996). Physiological origins and functional correlates of EEG rhythmic activities: Implications for self-regulation. Biofeedback and Self-regulation, 21, 3-33. http://doi.org/10.1007/BF02214147.

Tremayne, P., \& Barry, R. J. (2001). Elite pistol shooters: Physiological patterning of best vs. worst shots. International Journal of Psychophysiology, 41, 19-29. http:/ doi.org/10.1016/S0167-8760(00)00175-6.

Von Stein, A., \& Sarnthein, J. (2000). Different frequencies for different scales of cortical integration: From local gamma to long range alpha/theta synchronization. International Journal of Psychophysiology, 38, 301-313. http://doi.org/10. 1016/S0167-8760(00)00172-0.

Zhu, F. F., Poolton, J. M., Wilson, M. R., Maxwell, J. P., \& Masters, R. S. W. (2011). Neural co-activation as a yardstick of implicit motor learning and the propensity for conscious control of movement. Biological Psychology, 87, 66-73. http://doi.org/10.1016/j.biopsycho.2011.02.004. 